in vivo $34: 1085-1094(2020)$

doi:10.21873/invivo.11879

\title{
BRM270 Suppresses Cervical Cancer Stem Cell Characteristics and Progression by Inhibiting SOX2
}

\author{
NISANSALA CHANDIMALI ${ }^{1,2^{*}}$, HU-NAN SUN ${ }^{3 *}$, YANG HO PARK ${ }^{4}$ and TAEHO KWON ${ }^{5}$ \\ ${ }^{1}$ Immunotherapy Convergence Research Center, Korea Research Institute \\ of Bioscience and Biotechnology (KRIBB), Daejeon, Republic of Korea; \\ ${ }^{2}$ Department of Functional Genomics, University of Science \& Technology, Daejeon, Republic of Korea; \\ ${ }^{3}$ College of Life Science \& Technology, Heilongjiang Bayi Agricultural University, Daqing, P.R. China; \\ ${ }^{4}$ Park Yang Ho BRM Institute, Seoul, Republic of Korea; \\ ${ }^{5}$ Primate Resources Center, Korea Research Institute of Bioscience \\ and Biotechnology (KRIBB), Jeonbuk, Republic of Korea
}

\begin{abstract}
Background/Aim: Cervical cancer is one of the leading causes of cancer death in women worldwide. BRM270 (BRMLife) has therapeutic potential for cancer treatment owing to its ability to inhibit cell proliferation, and expression of cluster of differentiation (CD) 133 in CD133+ cancer cells. This study was designed to evaluate the therapeutic effects of plant extract formulation BRM270 against cervical cancer progression. Materials and Methods: The expression of sex-determining region Y-box 2 (SOX2) was tested in four different cervical cancer cell lines, HeLA, SiHa, Caski and C33A. SOX2-expressing SiHa and C33A cell lines were selected for further experiments on the in vitro and in vivo effects of BRM270 on cervical cancer progression using western blotting, flow cytometry, sphereformation assay, magnetic-activated cell sorting of $C D 133^{+}$ cervical cancer cells, and xenografts in female athymic $B A L B / c$ nude mice. Results: In the present study, in cervical cancer stem cells (CSCs), we found that BRM270 inhibited expression of $S O X 2$, which is associated with cervical cancer initiation and metastasis. BRM270 also inhibited CD133 expression and induced apoptosis of CSCs and suppressed CD133 ${ }^{+}$CSC proliferation and sphere formation in vitro as
\end{abstract}

This article is freely accessible online.

*These Authors contributed equally to this work.

Correspondence to: Taeho Kwon, Primate Resources Center, Korea Research Institute of Bioscience and Biotechnology (KRIBB), 35133 Neongme-gil, Ibam-myeon, Jeongeup-si, Jeonbuk, 56216, Republic of Korea. Tel: +82 635705316, Fax: +82 635705309, e-mail:kwon@kribb.re.kr

Key Words: BRM270, cervical cancer, cancer stem cells, epithelialto-mesenchymal transition, SOX2. well as SiHa and C33A cell xenograft tumor growth in vivo. This was accompanied by down-regulation of markers of epithelial-to-mesenchymal transition. Conclusion: BRM270 might be an effective agent for cervical cancer treatment.

Cervical cancer is the most common malignancy and leading cause of cancer-associated mortality in females worldwide (1). Most women with this disease are diagnosed at late stages and have poor prognosis (2). Human papillomavirus (HPV) infection and genetic as well as other factors are thought to play an important role in the development of cervical cancer (3). In particular, oncogenic HPV types promote the development of cervical cancer precursor lesions (4). However, only a small fraction of HPV-infected females develops the disease, indicating that other factors contribute to its genesis (5).

Cervical cancer stem cells (CSCs), which generate multiple types of tumor cell and are associated with metastasis and tumor recurrence, are potential therapeutic targets for the treatment of cervical cancer (6). CSC markers such as aldehyde dehydrogenase (1), cluster of differentiation 133 (CD133), and sex-determining region Y-box 2 (SOX2) were identified as being specific to CSCs in cervical cancer cell lines (6). SOX genes encode high-mobility group transcription factors that play critical roles in organogenesis (7). The tissue specificity and function of SOX proteins are largely determined by their association with other transcriptional regulators (7). SOX2 has recently been implicated in human cancer; for example, its expression is linked to a stem cell-like state in cervical cancer (8). SOX2 has also been shown to regulate tumor initiation and CSC functions (9). There are chemotherapeutic drugs, such as 5-fluorouracil and gemcitabine, for cervical cancer but acquired resistance to those drugs after several cycles of therapy remains a major challenge to overcome for successful management of cervical cancer (10). 
Herbal medicines are naturally occurring, plant-derived substances used to treat illnesses (11). These natural herbal compounds isolated from fruits and vegetables such as cinnamon, and grape seed proanthocyanidins, have been observed to have potential chemotherapeutic effects against various cancer types including cervical cancer $(12,13)$. BRM270, a formulation of extracts from seven Asian plants (Saururus chinensis, Citrus unshiu Markovich, Aloe vera, Arnebia euchroma, Portulaca oleracea, P. vulgaris var. lilacina, and Scutellaria baicalensis; Table I) (14), has bioactivity against nuclear factor (NF)-kB and was shown to inhibit cancer cell growth in vitro $(14,15)$. Moreover, BRM270 has shown therapeutic potential for the treatment of osteosarcoma, which was associated with the suppression of cell proliferation and cell-cycle progression, as well as CD133 expression (14).

In the present study, we investigated whether BRM270 has similar effects on cervical cancer.

\section{Materials and Methods}

BRM270 formulation. The herbal ingredients of BRM270 were obtained from Park Yang Ho BRM Institute (Seoul, Republic of Korea) and kindly authenticated by KIPRIS (Korea Intellectual Property Rights Information Service, BRM270: BRMLife, Patent Registration No.10-1794080; http://engportal.kipris.or.kr/engportal/ search/total_search.do).

Extraction of BRM270 and constituent herbs. The ethanolic extract was prepared as follows: The dried and pulverized medicinal herbs were mixed together and a 1-kg batch was soaked with $40 \%$ ethanol (4 1 for $24 \mathrm{~h}$ ). The ethanolic extract was concentrated with a rotary evaporator, and lyophilized, and reconstituted in dimethysulfoxide (DMSO) for the in vitro studies. We previously determined the proportions (w/w) of these herbs in BRM270 (14).

Cell culture. HeLa, CaSKi, $\mathrm{SiHa}$, and C33A human cervical cancer cell lines were obtained from the American Type Culture Collection (Manassas, VA, USA). HeLa, CaSKi, SiHa, and C33A cells were maintained in Dulbecco's modified Eagle's medium (DMEM; Invitrogen, Carlsbad, CA, USA) containing 10\% fetal bovine serum (Hyclone, Logan, UT, USA), penicillin (100 U/ml, Hyclone), and streptomycin (100 mg/ml; Hyclone) (16). Endogenous SOX2 expression was determined in each cell line as described below.

Cell viability assay. Cells were seeded at a density of $4 \times 10^{4}$ cells/well in a 96-well plate and the effects of BRM270 at different concentrations $(10,20,40,60,80,100$, and $150 \mu \mathrm{g} / \mathrm{ml})$ for $48 \mathrm{~h}$ on cervical cancer cell proliferation was studied using EZ-Cytox kit (Dogenbio, Seoul, Korea) according to the manufacturer's instructions after the cells were treated with BRM270 for $48 \mathrm{~h}$. The optical density (OD) of each well was measured at $450 \mathrm{~nm}$ by using a scanning multi-well spectrophotometer. BRM270 at $80 \mu \mathrm{g} / \mathrm{ml}$ for $48 \mathrm{~h}$ was selected as the treatment concentration for further experiments.

Western blotting. Protein samples which were isolated from $\mathrm{SiHa}$, C33A, HeLa and CaSKi cell lines, were separated by $10 \%$ or $12 \%$ sodium dodecyl sulfate polyacrylamide gel electrophoresis and
Table I. The composition of BRM270.

\begin{tabular}{lcc}
\hline Species name & Country of origin & \% By dry mass \\
\hline Saururus chinensis & Korea & 36.0 \\
Citrus unshiu Markovich & Korea & 24.2 \\
Portulaca oleracea & Korea & 10.6 \\
P. vulgaris var. lilacina & Korea & 6.2 \\
Scutellaria baicalensis & China & 10.6 \\
Arnebia euchroma & Korea & 6.2 \\
Aloe vera & America & 6.2 \\
Total amount & & 100 \\
\hline
\end{tabular}

transferred to a nitrocellulose membrane that was probed with primary antibodies against apoptotic peptidase activating factor 1 (APAF1), NF-kB, BCL2 apoptosis regulator (BCL2), caspase-3, cleaved caspase-3, Nanog homeobox (NANOG), SOX2, snail family transcriptional repressor 1 (SNAI1), E-cadherin, and vimentin (all from Santa Cruz Biotechnology, Dallas, TX, USA); POU class 5 homeobox 1 (OCT3/4) (Abcam, Cambridge, MA, USA); and glyceraldehyde 3-phosphate dehydrogenase (Abfrontier, Seoul, Korea). Western blotting was performed using the iBlot $2 \mathrm{Gel}$ Transfer system (Thermo Fisher Scientific, Waltham, MA, USA).

Flow cytometry. To evaluate apoptosis after 48-h treatment with BRM270 at $80 \mu \mathrm{g} / \mathrm{ml}$, SOX2-expressing SiHa and C33A cells were washed with phosphate-buffered saline (PBS), stained with Annexin V Binding Buffer (BD Biosciences, San Diego, CA, USA), and labeled with fluorescein isothiocyanate-conjugated Annexin V (BD Biosciences) according to the manufacturer's protocol. Cells were sorted on a FACS Calibur flow cytometer (BD Biosciences) (17).

Wound-healing and invasion assays. SOX2-expressing $\mathrm{SiHa}$ and C33A cells were trypsinized and seeded in 96-well tissue culture plates and reached $100 \%$ confluence after $48 \mathrm{~h}$. A scratch was made in the cell monolayer using a sterile 96-Well Wound Maker (Essen Bioscience, Winooski, VT, USA). Cells were washed twice with PBS and cultured in DMEM containing $10 \%$ fetal bovine serum. Images of $\mathrm{SiHa}$ and $\mathrm{C} 33 \mathrm{~A}$ cells migrating into the wound area were acquired at 0 and $48 \mathrm{~h}$ with an inverted IncuCyte ZOOM Live-Cell Analysis system (Essen Bioscience). The invasion assay was performed using a transwell chamber consisting of 8-m membrane filter inserts (Corning Inc., Corning, NY, USA) coated with Matrigel (Sigma-Aldrich, St. Louis, MO, USA). Briefly, cells were trypsinized and resuspended in serum-free medium; $1 \times 10^{5}$ cells were added to the upper chamber, whereas the lower chamber was filled with medium containing $10 \%$ fetal bovine serum. After incubating the cells for $24-48 \mathrm{~h}$, cells that passed through the coated membrane to the lower surface were fixed with $4 \%$ paraformaldehyde and stained with hematoxylin. Cells were counted under a light microscope at $100 \times$ magnification.

Sphere formation assay. SOX2-expressing SiHa and C33A cells $\left(2 \times 10^{3} /\right.$ well $)$ were seeded in 6-well Ultra Low Cluster plates (Corning Inc.) and cultured in suspension in serum-free DMEM/F12 (Gibco, Grand Island, NY, USA) containing B27 supplement (1:50; Invitrogen), $20 \mathrm{ng} / \mathrm{ml}$ epidermal growth factor (Calbiochem, San Diego, CA, USA), and $0.5 \%$ bovine serum albumin (Sigma-Aldrich) 
for 10-14 days. The number of $\mathrm{SiHa}$ and C33A cell spheres (tight, spherical, non-adherent masses $>100 \mu \mathrm{m}$ in diameter) was counted, and images of the spheres were acquired with an inverse microscope. Sphere-formation efficiency was calculated as colonies/input cells $\times 100 \%$ (17).

Sphere immunocytochemistry. $\mathrm{CD}_{133^{+}}$and $\mathrm{CD} 133^{-}$cells were harvested with gentle trypsinization, washed and resuspended with serum-free DMEM/F12 (Gibco, Grand Island, NY, USA) containing B27 supplement (1:50; Invitrogen), 20ng/ml epidermal growth factor (Calbiochem, San Diego, CA, USA), and $0.5 \%$ bovine serum albumin (Sigma-Aldrich). Single cells were confirmed under a microscope, counted and $4 \times 10^{3}$ cells/well seeded in 96-well Ultra Low Cluster plates (Corning Inc.) for 3 days. Spheres were then fixed $30 \mathrm{~min}$ with $30.03 \mathrm{~g} / \mathrm{mol}$ formaldehyde solution. Cells were then rinsed twice with PBS and incubated in blocking solution consisting of $1 \times$ PBST with $1 \%$ bovine serum albumin for $1 \mathrm{~h}$. Cells were incubated overnight at $4{ }^{\circ} \mathrm{C}$ with primary antibody to SOX2 and CD133 from Santa Cruz Biotechnology) with a solution consisting of $0.1 \%$ Triton-X100,10\% $\mathrm{NaNO}_{3}$ and $1 \times \mathrm{PBS}$. Cells were rinsed twice in $1 \times$ PBST prior to incubating with secondary antibody for 2 $\mathrm{h}$ in the dark at room temperature. Cells were then rinsed twice with $1 \times$ PBST and counterstained with diluted in $1 \times$ PBS for 20 min prior to visualization and image capturing using microscopy.

Animals. In the present study, 8-week-old female athymic BALB/c nude mice were used for in vivo experiments. The animals were provided by Central Laboratory Animal Resources, Korea Research Institute of Bioscience \& Biotechnology (KRIBB), Daejeon, Republic of Korea. The animals were kept in polypropylene cages in a room with controlled temperature $\left(22^{\circ} \mathrm{C} \pm 1\right), 60-70 \%$ humidity and a $12 \mathrm{~h} \mathrm{light} / 12 \mathrm{~h}$ dark cycle and provided with standard food pellets and drinking water ad libitum, in Central Laboratory Animal Resources, KRIBB, Daejeon, Korea. The animals were divided randomly into groups and kept under observation throughout the duration of experimentation, in terms of body weight, food and water consumption, and for any sign of health toxicity. At the end of the experiments, all the mice were euthanized by $\mathrm{CO}_{2}$ asphyxiation in a $\mathrm{CO}_{2}$ chamber.

The experiments were approved by the Government of Korea and Institutional Animal Care and Use Committee-approved protocols (IACUC code No. KRIBB-AEC-16208) of KRIBB. The experiments were carried out as per their guidelines.

Xenograft model. The tumorigenicity of $\mathrm{SiHa}$ and $\mathrm{C} 33 \mathrm{~A}$ cells was determined by subcutaneous inoculation of $1 \times 10^{5}$ cells resuspended in $100 \mu \mathrm{l}$ Matrigel (Sigma-Aldrich) in PBS into the flanks of 8week-old female athymic BALB/c nude mice (five per group). $\mathrm{BALB} / \mathrm{c}$ nude mice with subcutaneously inoculated $\mathrm{SiHa}$ and C33A cells were divided into two groups $(n=5)$. BRM270-treated and untreated $\mathrm{SiHa}$ and $\mathrm{C} 33 \mathrm{~A}$ cells in Matrigel were implanted into mice to generate tumors at an orthotopic site that provided a suitable environment for tumor growth. The first group, which served as a control group, did not receive BRM270, while the second group received $1 \mathrm{mg} / \mathrm{kg}$ of BRM270 in a daily manner. BRM270 was administrated to mice via oral gavage which was undertaken using a feeding catheter (C1 LifeTECH, Osong, Korea). Tumor size was measured using calipers (volume $=$ shortest diameter ${ }^{2} \times$ longest diameter/2) every 3 days. Grafts were removed 50 days after cell inoculation and photographed (17).
MACS of $\mathrm{CD} 33^{+}$cervical cancer cells. SiHa and C33A cells were labeled with a primary antibody to CD133 (Miltenyi Biotec, Auburn, CA, USA), magnetically labeled with rat anti-mouse IgG micro beads (Miltenyi Biotec), and separated on a MACS liquid separation column (Miltenyi Biotec) according to the manufacturer's instructions. The purity of sorted cells was evaluated by western blotting.

Statistical analysis. Data were analyzed using SPSS v.20.0.1 software (IBM, Armonk, NY, USA). Differences between groups were evaluated with the chi-squared test or Fisher's exact test as appropriate. Values of $p<0.05$ were considered statistically significant.

\section{Results}

BRM270 inhibits SOX2 expression and reduces viability in cervical cancer cells. SOX2 is a marker that has been implicated in cervical cancer development and is therefore a potential therapeutic target (18). We investigated whether SOX2 is expressed in cervical cancer by western blotting using HPV-positive and -negative human cervical cancer cell lines. SOX2 was not expressed in HeLa or CaSKi cells (Figure 1A). When SOX2-expressing HPV-positive SiHa and HPV-negative C33A cells were treated with different concentrations $(10,20,40,60,80,100$, and $150 \mu \mathrm{g} / \mathrm{ml})$ of BRM270 for $48 \mathrm{~h}$, cell viability was inhibited in both cell lines in a dose-dependent manner (Figure 1B). BRM270 at $80 \mu \mathrm{g} / \mathrm{ml}$ was selected as the treatment concentration for further experiments.

BRM270 treatment inhibited the expression of SOX2 (Figure 1C and D), the pro-apoptotic protein APAF1, and antiapoptotic proteins NF-KB and BCL2 but increased that of caspase-3 (Figure 1E). Moreover, BRM270 treatment induced apoptosis of $\mathrm{SiHa}$ and $\mathrm{C} 33 \mathrm{~A}$ cells (Figure 1F) and reduced colony formation in the clonogenic assay (Figure $1 \mathrm{G}$ ). These results suggest that BRM270 inhibits the development of cervical cancer by suppressing cell viability and promoting apoptosis via modulation of SOX2 expression.

BRM270 reduces cervical cancer CSC activity. We next investigated whether BRM270 influences cervical cancer cell migration and invasion. BRM270 prevented wound closure by $\mathrm{SiHa}$ and $\mathrm{C} 33 \mathrm{~A}$ cells (Figure 2A) and inhibited their invasive capacity (Figure 2B). These data indicate that BRM270 suppresses motility of cervical cancer cells, which is a property associated with metastatic progression.

CSCs are characterized by their sphere-forming ability in vitro; sphere formation assays under serum-free culture conditions are therefore widely used to isolate and enrich CSCs (19). We investigated whether BRM270 had inhibitory effects on cervical cancer CSCs by treating SiHa and C33A cells with different concentrations of the formulation. The optimal concentration was determined as $80 \mu \mathrm{g} / \mathrm{ml}$ this was used in subsequent experiments. BRM270 inhibited sphere 
A

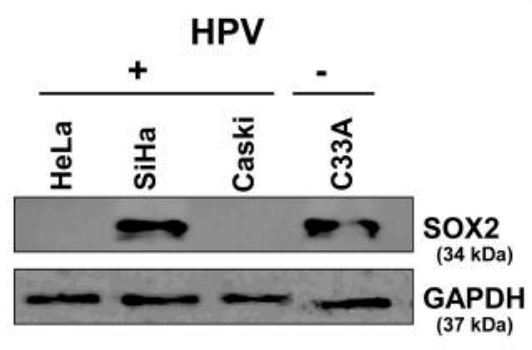

B

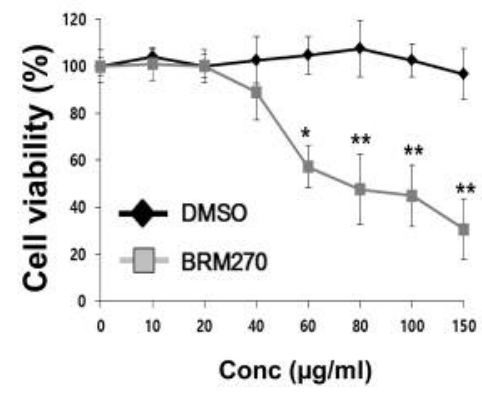

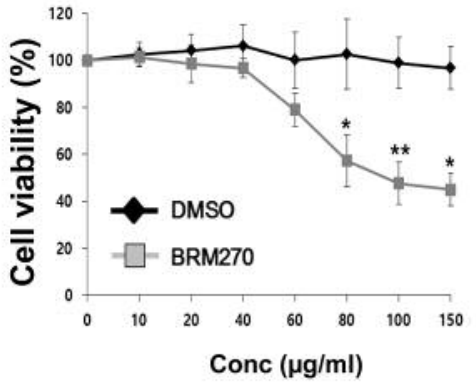

C
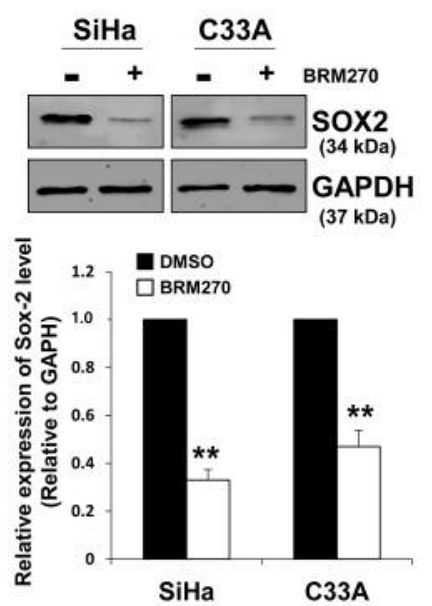

F

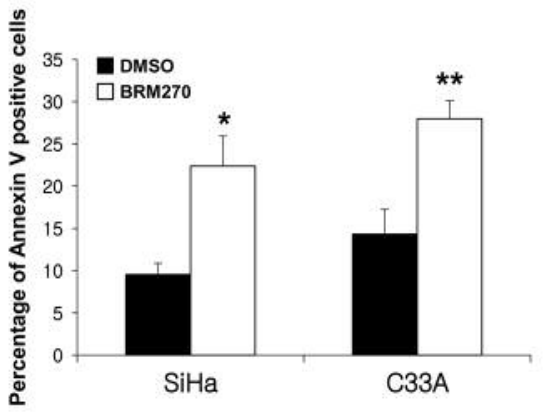

D

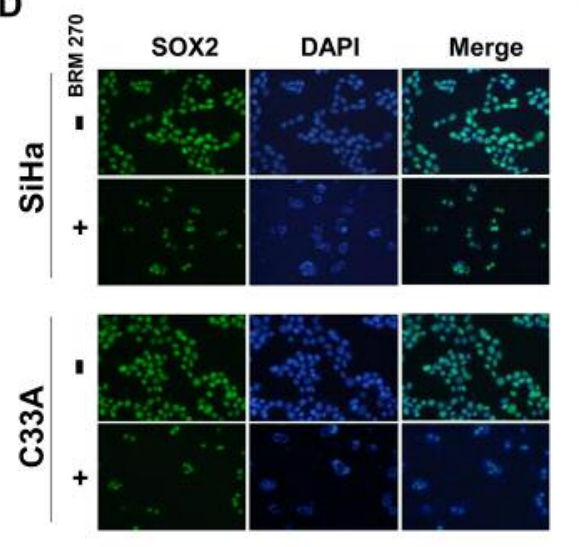

G

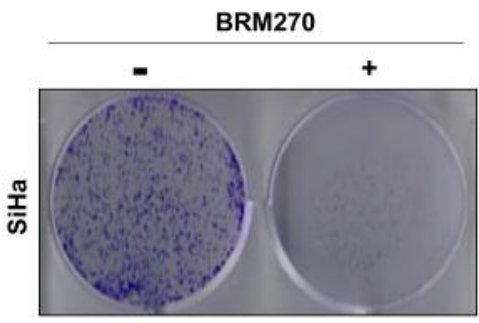

E

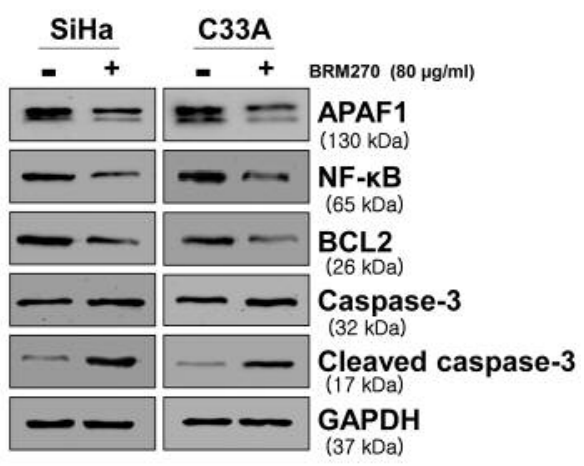

Figure 1. BRM270 inhibits cervical cancer cell proliferation. A: Sex-determining region Y-box 2 (SOX2) protein expression was detected by western blotting. GAPDH: Glyceraldehyde 3-phosphate dehydrogenase. B: SiHa and C33A cell proliferation was evaluated by cell viability assay after 48 h. C: SOX2 expression in SiHa and C33A cells treated with BRM270. DMSO: Dimethyl sulfoxide. D: Representative images of SiHa and C33A cells treated with BRM270 at $80 \mu \mathrm{g} / \mathrm{ml}$ for $48 \mathrm{~h}$ and labeled with an antibody against SOX2. E: Expression of apoptosis-associated proteins apoptotic peptidase activating factor 1 (APAF1), nuclear factor (NF)-kB, BCL2 apoptosis regulator (BCL2), caspase-3 and cleaved caspase-3, in cells treated with BRM270 at $80 \mu \mathrm{g} / \mathrm{ml}$ for $48 \mathrm{~h}$. F: Apoptosis of BRM270-treated SiHa and C33A cells as assessed by flow cytometry. G: Colony formation by SiHa and C33A cells treated with BRM270 at $80 \mu \mathrm{g} / \mathrm{ml}$ for $48 \mathrm{~h}$ was evaluated by clonogenic assay. Data represent the mean \pm SEM $(n=5 \mathrm{per}$ group). Significantly different at $* p<0.05$, and $* * p<0.01$.

formation and SOX2 and CD133 expression in both cell lines in a concentration-dependent manner, as determined by immunofluorescence analysis (Figure $2 \mathrm{C}$ and $\mathrm{D}$ ). Moreover, protein levels of the CSC markers OCT3/4 and NANOG were down-regulated by BRM270 treatment (Figure 2E). These results provide additional evidence that BRM270 blocks cervical cancer progression by inhibiting the expression of stemness genes. 
A
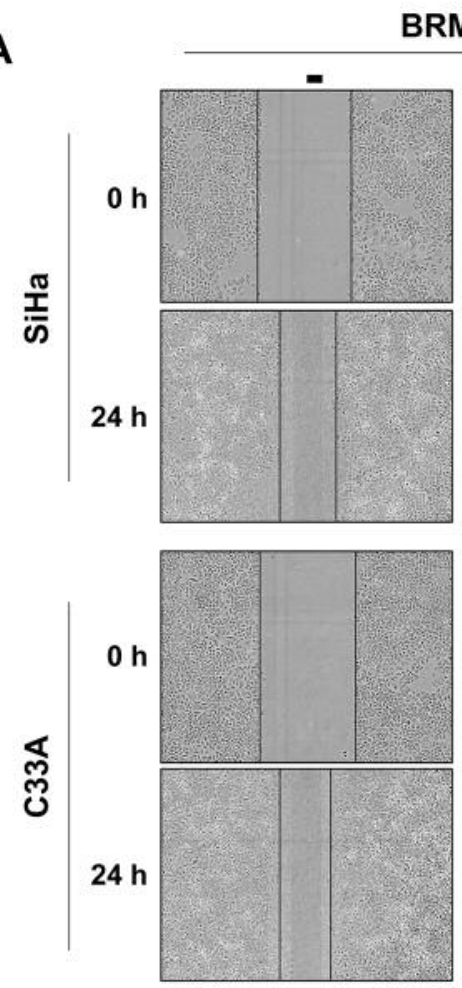

C

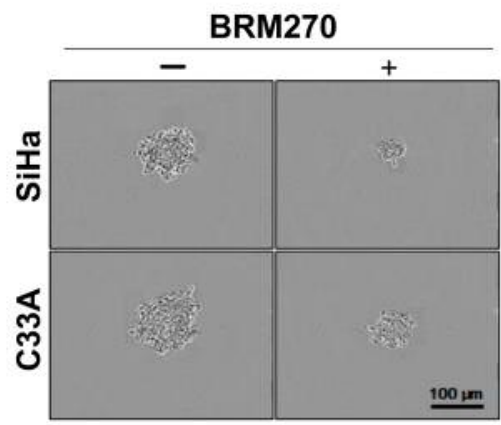

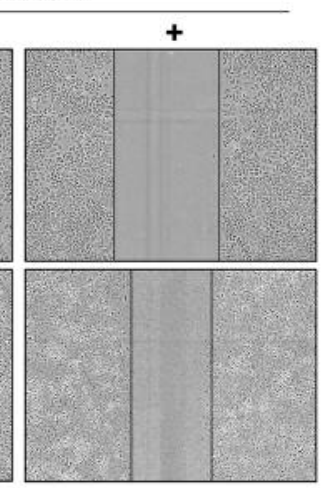

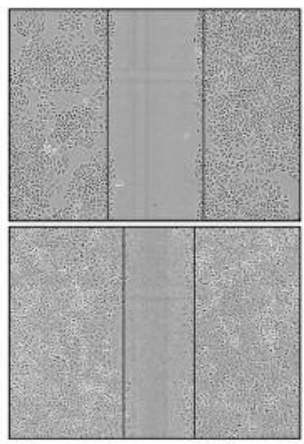

B
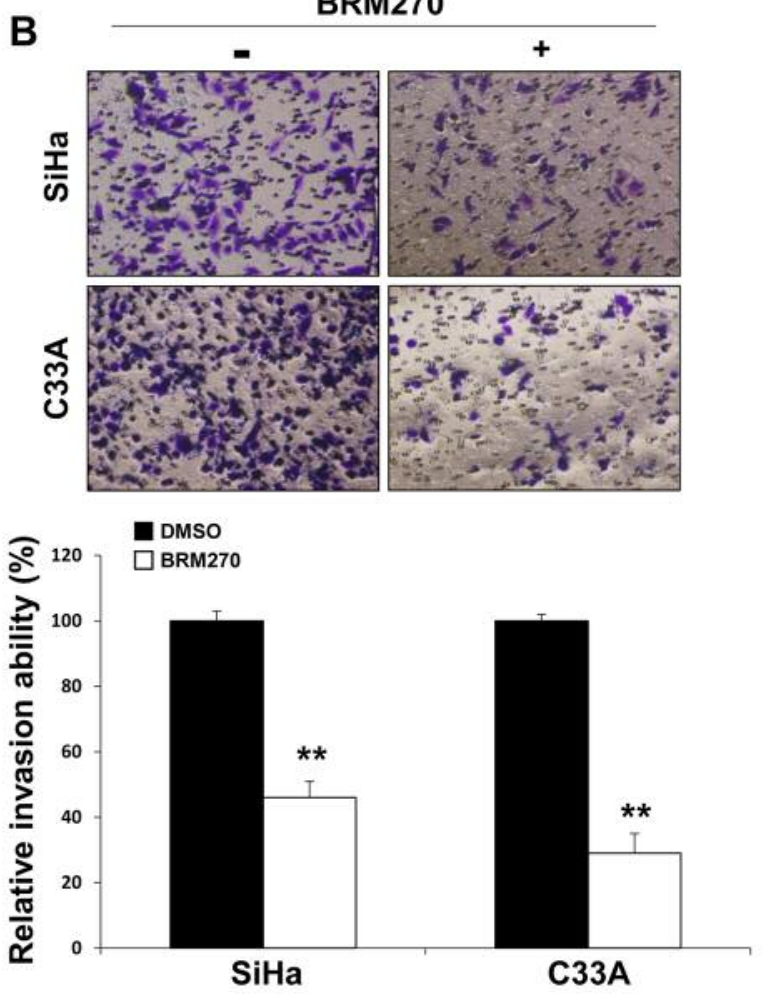

D

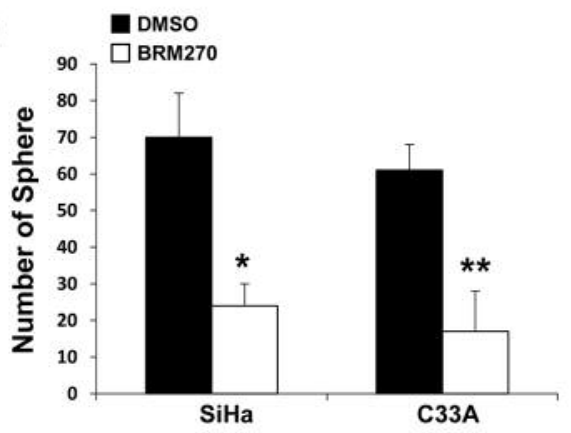

E
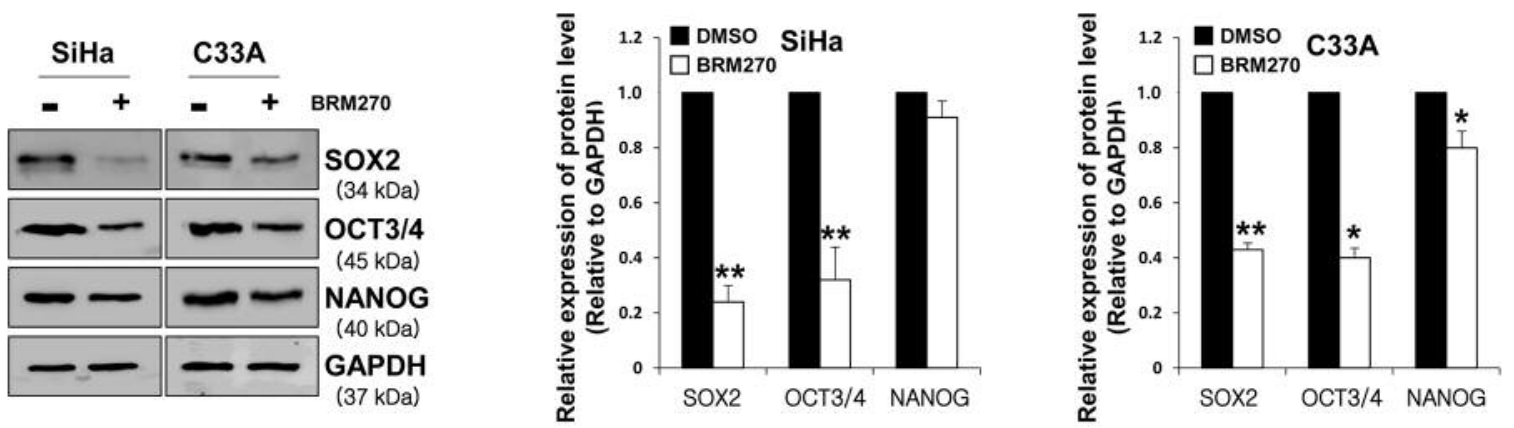

Figure 2. BRM270 suppresses cervical cancer stem cell (CSC) characteristics. A: SiHa and C33A cell migration was assessed with the woundhealing assay $48 \mathrm{~h}$ after treatment with BRM270 $(80 \mu \mathrm{g} / \mathrm{ml})$ or vehicle dimethyl sulfoxide (DMSO). B: Invasion was evaluated with transwell assay 48 h after treatment with BRM270 or vehicle. C: Representative images of SiHa and C33A sphere-forming cells. Bar=100 $\mu \mathrm{m}$. D: The number of spheres was counted and normalized to that of the control group. E: Western blot analysis of CSC marker expression. SOX2: Sex-determining region Y-box 2; OCT3: POU class 5 homeobox 1; NANOG: Nanog homeobox; GAPDH: glyceraldehyde 3-phosphate dehydrogenase. Data represent the mean $\pm \operatorname{SEM}$ ( $n=5$ per group). Significantly different at $* p<0.05$, and $* * p<0.01$. 
A

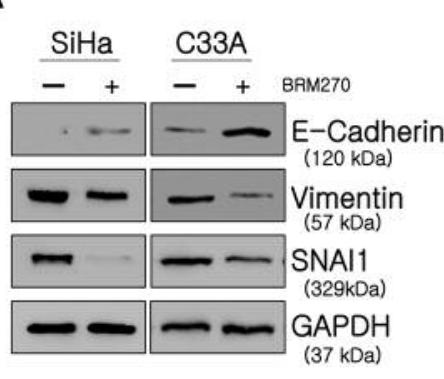

B

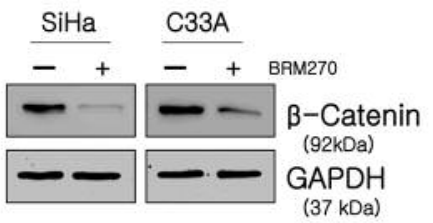

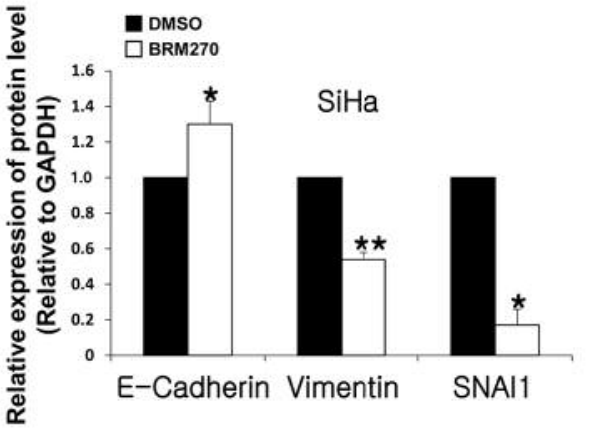

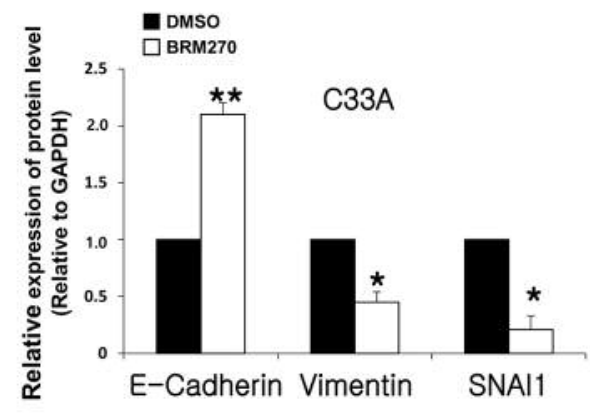

C

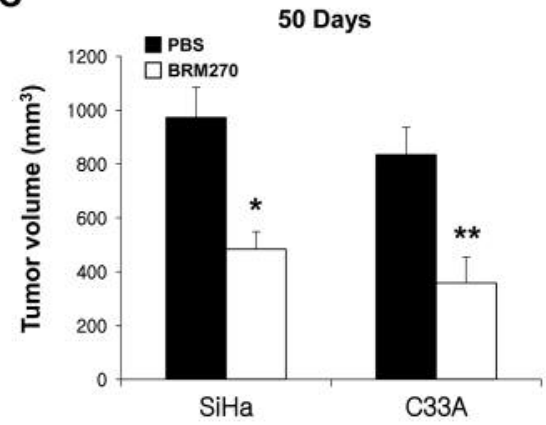

D

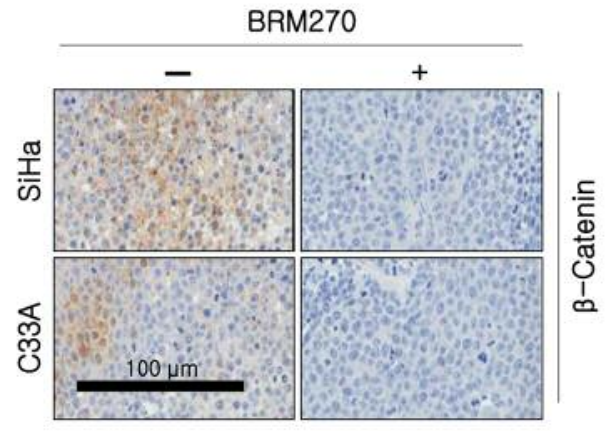

Figure 3. BRM270 inhibits $\beta$-catenin signaling in cervical cancer cells. Effect of BRM270 treatment on the expression of E-cadherin, vimentin, and snail family transcriptional repressor 1 (SNAI1) (A) and $\beta$-catenin (B) in SiHa and C33A cells, as determined by western blotting. GAPDH: Glyceraldehyde 3-phosphate dehydrogenase. C: Tumor volume was monitored for 50 days after injection of SiHa and C33A cells treated with BRM270 or untreated cells into nude mice. D: Immunohistochemical analysis of $\beta$-catenin expression in tumors derived from SiHa and C33A cells treated with BRM270 or left untreated (200x magnification). Bar $=100 \mu \mathrm{m}$. Data represent the mean $\pm S E M(n=5$ per group). Significantly different at $* p<0.05$, and $* * p<0.01$.

BRM270 suppresses expression of epithelial-to-mesenchymal (EMT) markers and $\beta$-catenin signaling in cervical cancer cells. EMT occurs during the initial stage of tumor metastasis and involves the loss of epithelial characteristics and cell polarity, and the acquisition of mesothelial characteristics that enable cancer cells to invade adjacent tissues (20). Indeed, expression of the epithelial markers Ecadherin and $\beta$-catenin is reportedly down-regulated in the early stages of cervical cancer progression (21). It was previously shown that BRM270 suppressed tumor properties such as migratory capacity (14), and we recently reported that BRM270 targets CSCs (14). To determine whether BRM270 inhibits EMT in cervical CSCs, SiHa and C33A cells were analyzed for expression of the EMT markers Ecadherin, vimentin, and SNAI1 following treatment. Vimentin and SNAI1 levels were down-regulated whereas that of E-cadherin was increased in $\mathrm{SiHa}$ and C33A cells by BRM270 treatment (Figure 3A). Since $\beta$-catenin is the effector of WNT signaling (21), we investigated whether BRM270 suppressed the WNT/ $\beta$-catenin signaling cascade by western blotting. BRM270 treatment reduced $\beta$-catenin expression in both cell lines (Figure 3B). 
A

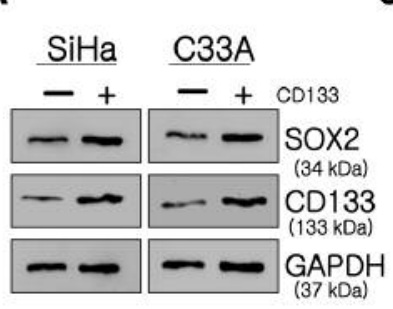

B

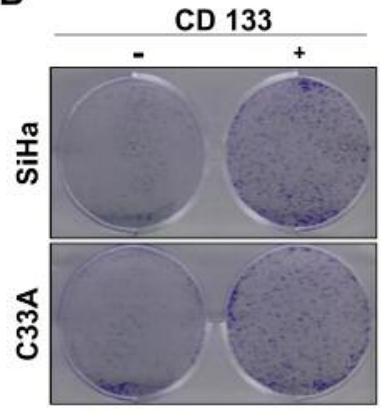

F

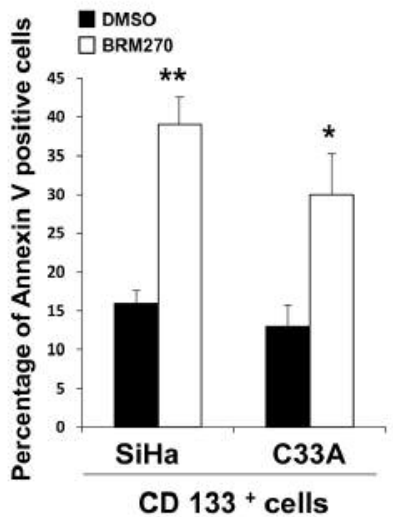

C

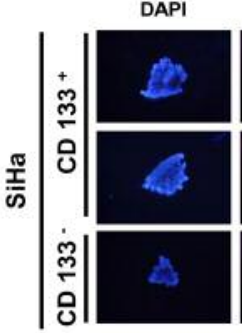

D

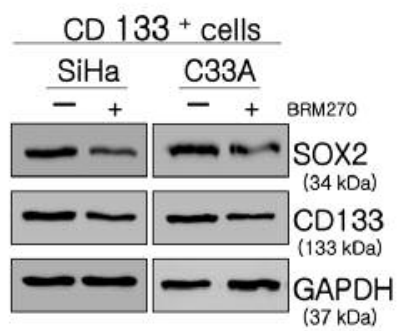

G
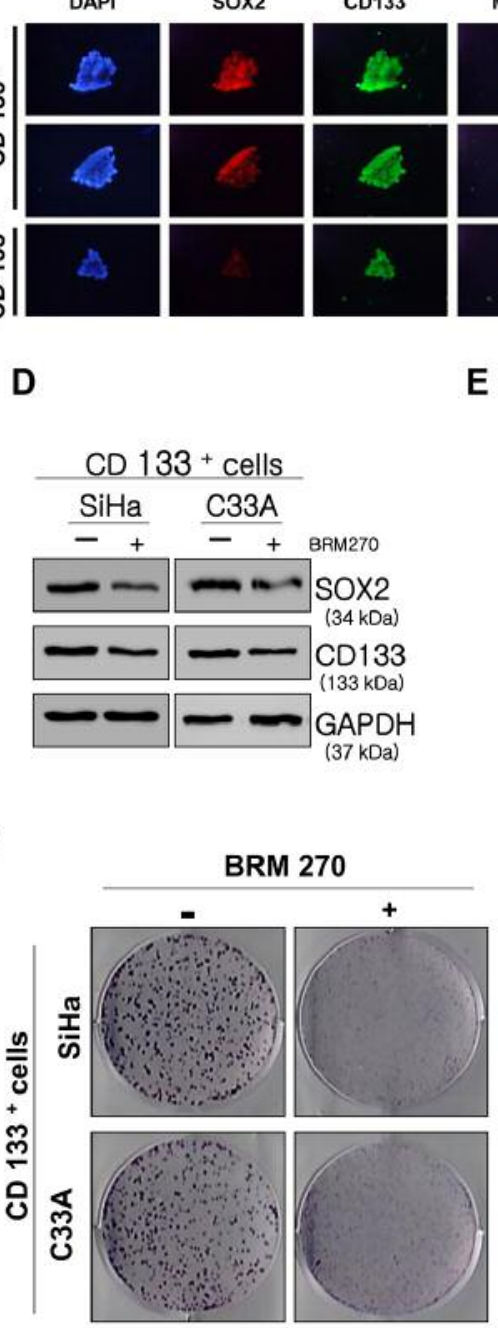

E
Merge
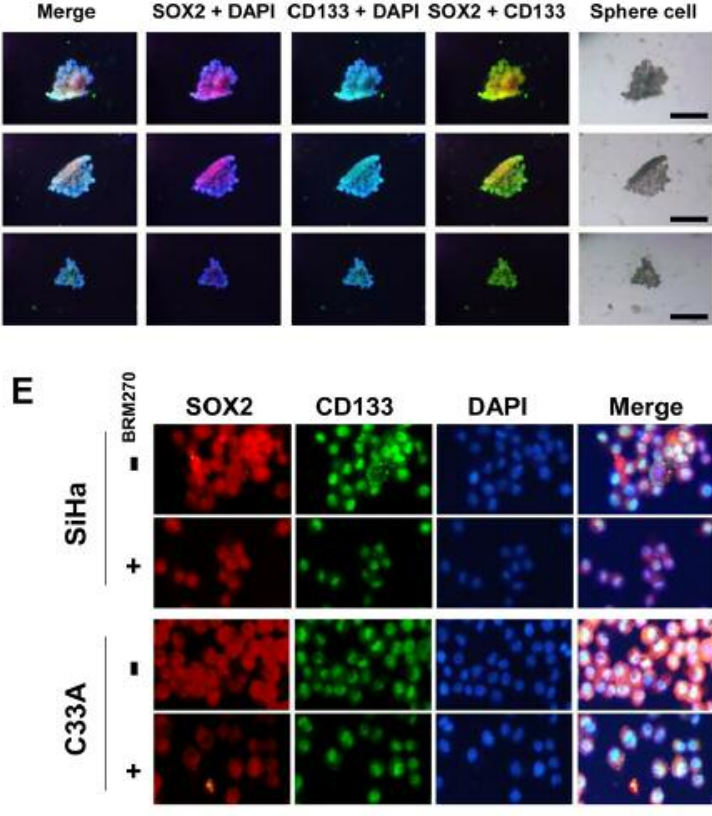

H

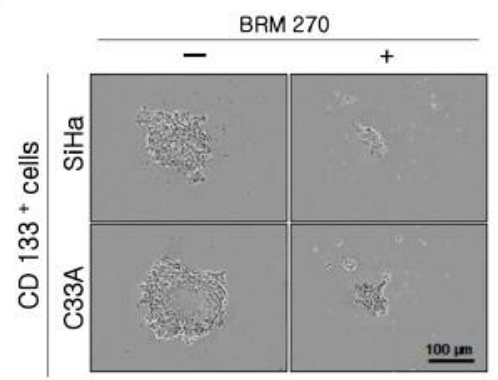

I

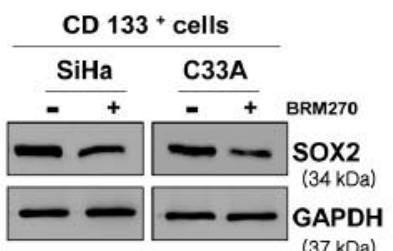

Figure 4. BRM270 suppresses expression sex-determining region Y-box 2 (SOX2) in CD133+ cervical cancer cells. A: SOX2 and CD133 expression in $\mathrm{CD} 133^{-}$and $\mathrm{CD} 133^{+}$cells, as detected by western blotting. GAPDH: Glyceraldehyde 3-phosphate dehydrogenase. B: Colony-formation capacity of $\mathrm{CD}_{133^{-}}$and $\mathrm{CD} 133^{+}$, as evaluated with the clonogenic assay. C: SOX2 was highly expressed in cancer stem cell $\left(C D 133^{+}\right)$spheres. Fluorescent immunocytochemistry was performed on SiHa CD133+ and CD133- sphere cells using monoclonal antibodies against SOX2 and CD133. Nuclear staining was performed using 4',6-diamidino-2-phenylindole (DAPI; blue). D: Expression of SOX2 in SiHa and C33A cells treated with BRM270 $(80 \mu \mathrm{g} / \mathrm{ml}$ for $48 \mathrm{~h})$ or left untreated. E: Representative images of CD133+ SiHa and C33A cells treated with BRM270 or left untreated, and labeled with antibodies against SOX2 and CD133. F: Apoptosis of BRM270-treated CD133+ SiHa and C33A cells detected by flow cytometry. G: Colony formation by $\mathrm{CD}^{133^{+}} \mathrm{SiH}$ a and C33A cells treated with BRM270 or left untreated, as determined with the clonogenic assay. H: Representative

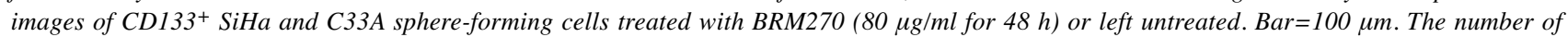
spheres was counted and normalized to that of the control group. I: SOX2 expression in SiHa and C33A CD133+ cell spheres treated with treated with BRM270 or left untreated. Data represent the mean \pm SEM ( $n=5$ per group). Significantly different at $* p<0.05$, and $* * p<0.01$.

We next investigated the effect of BRM270 on the tumorinitiating capacity of cervical cancer cell xenografts. BRM270 treatment inhibited cervical tumor initiation (Figure 3C), and an immunohistochemical analysis revealed that $\beta$-catenin immunoreactivity was reduced in tumors from BRM270-treated as compared to untreated $\mathrm{SiHa}$ and C33A cells (Figure 3D). 
A

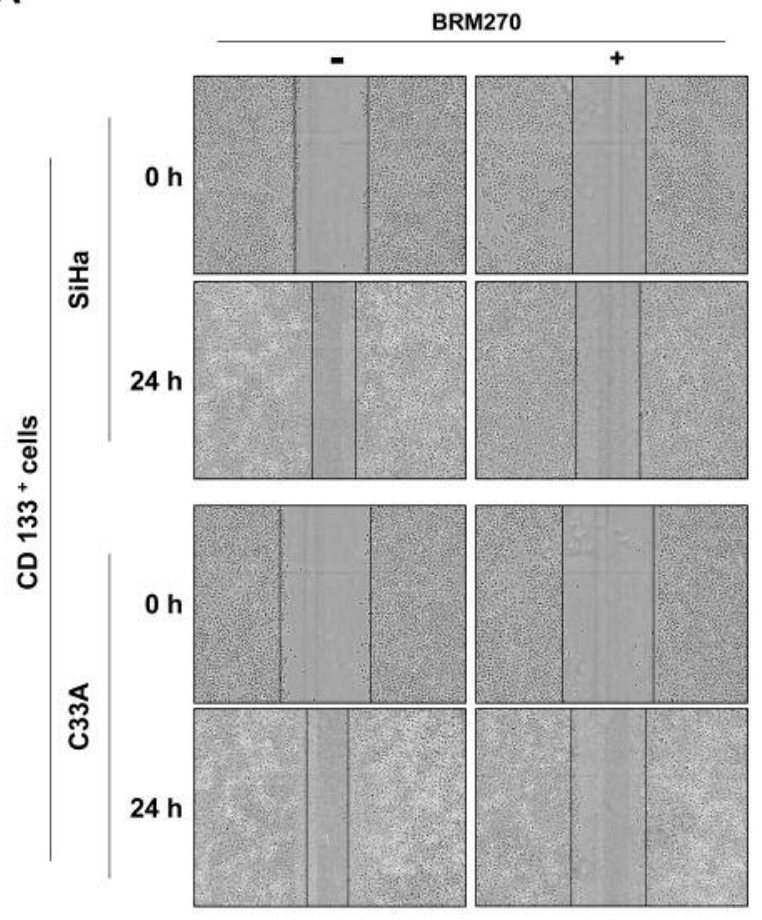

C

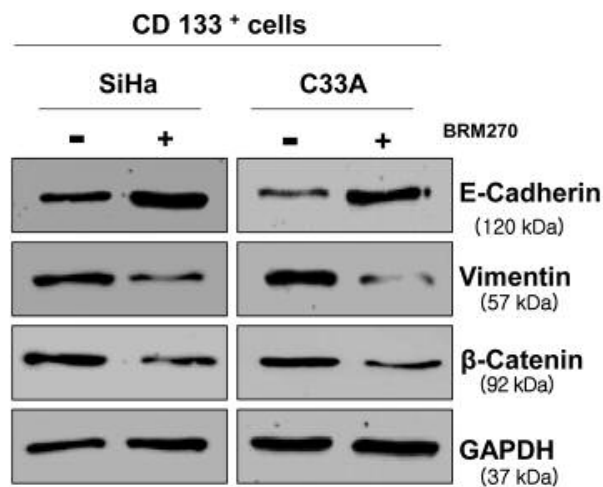

B
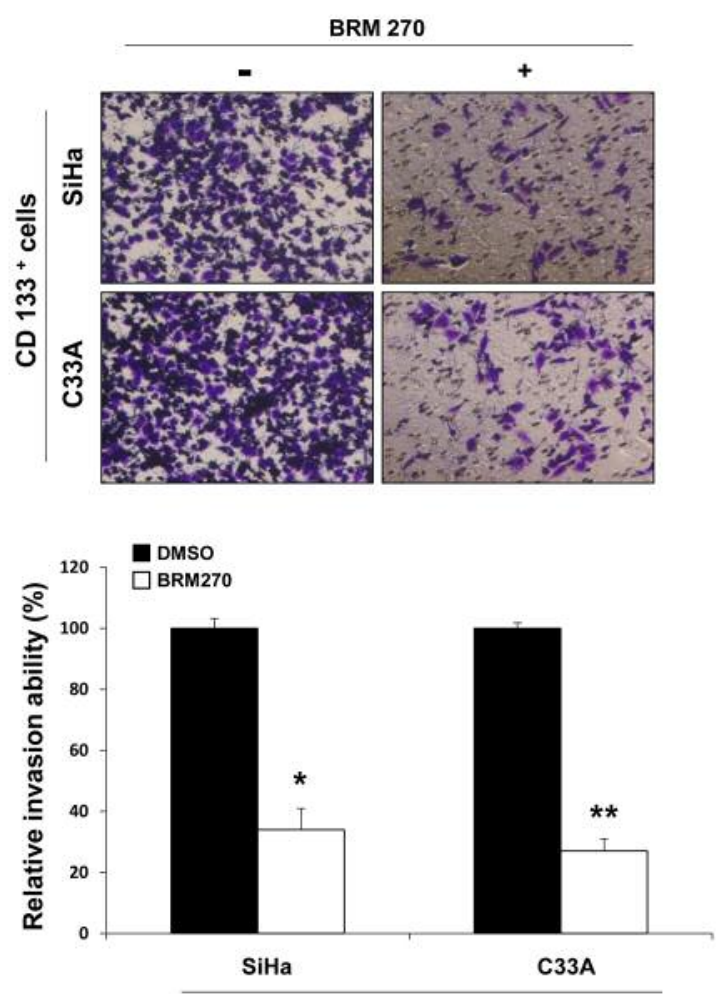

CD $133+$ cells

Figure 5. BRM270 inhibits migration, invasion, and $\beta$-catenin signaling in CD133+ cervical cancer cells. A: Migration of CD133+ SiHa and C33A cells was evaluated with the wound-healing assay $48 \mathrm{~h}$ after BRM270 (80 $\mu \mathrm{g} / \mathrm{ml}$ for $48 \mathrm{~h})$ or vehicle treatment. B: Invasion of BRM270-treated or untreated $\mathrm{CD}_{133^{+}} \mathrm{SiHa}$ and C33A cells was assessed with the transwell assay. C: E-Cadherin, vimentin, and $\beta$-catenin levels in SiHa and C33A

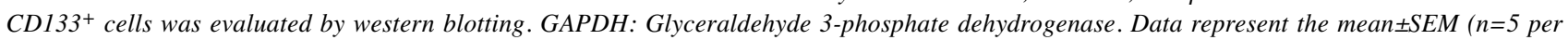
group). Significantly different at $* p<0.05$, and $* * p<0.01$.

BRM270 inhibits SOX2 expression and sphere-forming capacity in $\mathrm{CD}_{133^{+}}$cervical cancer cells. $\mathrm{CD}_{133^{+}}$cells exhibit stem cell-like properties such as self-renewal capacity, differentiation potential, enhanced proliferation, and tumorigenicity (22). To investigate whether BRM270 affected the properties of cervical CSCs, we separated CD133- from $\mathrm{CD} 133^{+} \mathrm{SiHa}$ and C33A cells by MACS (Figure 4A). SOX2 expression was evaluated in the two cell populations and it was found that it was higher in $\mathrm{CD}_{133^{+}}$than in $\mathrm{CD}_{133^{-}}$cells.
Accordingly, the colony-formation rate was also higher in $\mathrm{CD}_{133}{ }^{+}$than in $\mathrm{CD}_{133^{-}}$cells (Figure 4B). Next, we investigated the expression levels of SOX2 and CD133 in SiHa $\mathrm{CD}_{133^{+}}$and $\mathrm{CD}_{133^{-}}$sphere cells by immunocytochemistry. SOX2 was overexpressed in $\mathrm{CD}_{133^{+}}$sphere cells and there was very little expression of SOX 2 in $\mathrm{CD} 133^{-}$sphere cells. CD133 was also expressed highly in CD133+ ${ }^{+}$sphere cells when compare with $\mathrm{CD} 133^{-}$sphere cells (Figure 4C). BRM270 treatment resulted in a down-regulation of SOX2 
and CD133 expression in $\mathrm{CD} 133^{+} \mathrm{SiHa}$ and $\mathrm{C} 33 \mathrm{~A}$ cells (Figure 4D and E). We also examined the effects of BRM270 on cervical CSC apoptosis by flow cytometry after annexin$\mathrm{V}$ staining (Figure 4F). BRM270 treatment increased apoptosis and suppressed colony formation (Figure 4G), as well as sphere-forming capacity, in $\mathrm{CD}_{133}{ }^{+} \mathrm{SiHa}$ and $\mathrm{C} 33 \mathrm{~A}$ cells relative to untreated $\mathrm{CD}_{133^{+}}$cells (Figure $4 \mathrm{H}$ ). In addition, BRM270 reduced SOX2 levels in SiHa and C33A spheres (Figure 4I). These results indicate that BRM270 suppresses the stem cell properties of cervical CSCs.

BRM270 inhibits cell motility and $\beta$-catenin signaling in $C D 133^{+}$cervical cancer cells. We investigated the effects of BRM 270 on the metastatic potential of cervical cancer cells by evaluating migration and invasion of $\mathrm{CD} 133^{+} \mathrm{SiHa}$ and C33A cells treated with BRM270. BRM270 prevented wound closure in the migration assay (Figure 5A) and suppressed cell invasion (Figure 5B). Furthermore, BRM270 treatment down-regulated the expression of vimentin and $\beta$ catenin while increasing that of E-cadherin in $\mathrm{CD}_{133}{ }^{+} \mathrm{SiHa}$ and C33A cells (Figure 5C). Thus, BRM270 blocks EMT and metastatic progression of cervical cancer cells.

\section{Discussion}

Cervical cancer has a high recurrence rate and risk of metastasis following conventional therapy, resulting in a high mortality rate (23). CSCs are promising targets for the treatment of cervical carcinogenesis, given their association with cervical cancer initiation and metastasis. CSCs are the cellular basis for the phenotypic and functional heterogeneity among various cancer types $(24,25)$ and exhibit self-renewal and multi-lineage differentiation capacities that allow tumor development and growth (26). Escaping programmed cell death is a prerequisite for any tumor-initiating cell and is a mechanism that is associated with chemotherapeutic resistance; as such, therapeutics that enhance apoptosis are promising as anticancer strategies (27). In the present study, we found that BRM270 down-regulated the expression of the pro-apoptotic protein APAF1 and the anti-apoptotic proteins NF-kB and BCL2; however, it also increased the levels of the apoptosis effector protein caspase- 3 in cervical cancer cell lines, highlighting its therapeutic potential for treating cervical cancer.

SOX2 has been shown to regulate the behavior of transformed cells in the skin as well as CSCs and provides a link between tumor initiation and progression (9). Since SOX2 is associated with diverse cancer types, targeting its downstream effectors may be a more effective approach to treating specific malignancies. We found here that BRM270 inhibited SOX2 expression in cervical cancer cells and CCSCs, which was associated with reduced proliferation and sphere formation in the latter. This suggests that BRM270 can inhibit cervical cancer progression by suppressing the stem-cell character of CSCs.
Metastasis is associated with the self-renewal capacity of CSCs, which are also known as tumor-initiating cells (28). Recent studies have demonstrated a molecular link between self-renewal and EMT, suggesting that the latter plays critical roles in both early and late stages of metastatic progression (28). We found that BRM270 suppressed cervical cancer xenograft growth, which was associated with inhibition of EMT. CD133 is a membrane glycoprotein that is a marker of poor prognosis in cancer and is specifically expressed by CSCs (22). BRM270 was shown to reduce CD133 expression in $\mathrm{CD}_{133^{+}}$cervical cancer cells, providing further evidence of its effectiveness for treating cervical cancer.

In conclusion, our findings indicate that BRM270 can inhibit cervical cancer progression via inhibition of SOX2 and suppression of the stem cell properties of CSCs. Although the results from the xenograft model were promising, additional studies in animal models are need to evaluate the in vivo efficacy and potential toxicity of BRM270.

\section{Conflicts of Interest}

The Authors declare that there are no conflicts of interest in regard to this study.

\section{Authors' Contributions}

T.K., N.C. and H.N.S. designed the performed experiments, analyzed data, and wrote the article. Y.H.P. provided BRMLife (BRM270) components (Park Yang Ho BRM Institute). T.K. designed and wrote the whole article. All Authors read and approved the final article.

\section{Acknowledgements}

This research was supported by Basic Science Research Program through the National Research Foundation of Korea (NRF) funded by the Ministry of Education (2017R1D1A1B03028188).

\section{References}

1 Jemal A, Bray F, Center MM, Ferlay J, Ward E and Forman D: Global cancer statistics. CA Cancer J Clin 61(2): 69-90, 2011. PMID: 30207593. DOI: 10.3322/caac.21492

2 Gaffney D, Small B, Kitchener H, Young Ryu S, Viswanathan A, Trimble T, Covens A, Wilailak S, Lertkhachonsuk AA, Sitathanee C, Mahantshetty U, Fisher B, Springer S, Pollatz T, Spiller A, Bacon $M$ and Jhingran A: Cervix cancer research network (CCRN): Improving access to cervix cancer trials on a global scale. Int J Gynecol Cancer 26(9): 1690-1693, 2016. PMID: 30814204. DOI: 10.1097/IGC.0000000000000823

3 Yang A, Farmer E, Wu TC and Hung CF: Perspectives for therapeutic HPV vaccine development. J Biomed Sci 23(1): 75, 2016. PMID: 27809842. DOI: 10.1186/s12929-016-0293-9

4 Tomaic V: Functional roles of E6 and E7 oncoproteins in HPVinduced malignancies at diverse anatomical sites. Cancers $8(10)$ : 95, 2016. PMID: 27775564. DOI: 10.3390/cancers 8100095 
5 Wang HL, Lu X, Yang X and Xu N: Association of MBL2 exon 1 polymorphisms with high-risk human papillomavirus infection and cervical cancers: A meta-analysis. Arch Gynecol Obstet 294(6): 1109-1116, 2016. PMID: 27619685. DOI: 10.1007/ s00404-016-4201-z

6 Huang R and Rofstad EK: Cancer stem cells (CSCs), cervical CSCs and targeted therapies. Oncotarget 8(21): 35351-35367, 2017. PMID: 27343550. DOI: 10.18632/oncotarget.10169

7 Leis O, Eguiara A, Lopez-Arribillaga E, Alberdi MJ, HernandezGarcia S, Elorriaga K, Pandiella A, Rezola R and Martin AG: SOX2 expression in breast tumours and activation in breast cancer stem cells. Oncogene 31(11): 1354-1365, 2012. PMID: 21822303. DOI: $10.1038 /$ onc.2011.338

8 Liu XF, Yang WT, Xu R, Liu JT and Zheng PS: Cervical cancer cells with positive SOX2 expression exhibit the properties of cancer stem cells. PLoS One 9(1): e87092, 2014. PMID: 24489842. DOI: 10.1371/journal.pone.0087092

9 Boumahdi S, Driessens G, Lapouge G, Rorive S, Nassar D, Le Mercier M, Delatte B, Caauwe A, Lenglez S, Nkusi E, Brohee S, Salmon I, Dubois C, del Marmol V, Fuks F, Beck B and Blanpain C: SOX2 controls tumour initiation and cancer stemcell functions in squamous-cell carcinoma. Nature 511(7508): 246-250, 2014. PMID: 24909994. DOI: 10.1038/nature13305

10 Lin G, Chen Q, Yu S, Lin S, Yao H, Ding Z, Chen S, Lin MC and Wang $X$ : Overexpression of human telomerase reverse transcriptase $C$-terminal polypeptide sensitizes HeLa cells to 5fluorouracil-induced growth inhibition and apoptosis. Mol Med Rep 9(1): 279-284, 2014. PMID: 24190362. DOI: 10.3892/ mmr.2013.1777

11 Tilburt JC and Kaptchuk TJ: Herbal medicine research and global health: An ethical analysis. Bull World Health Organ 86(8): 594599, 2008. PMID: 18797616. DOI: 10.2471/blt.07.042820

12 Koppikar SJ, Choudhari AS, Suryavanshi SA, Kumari S, Chattopadhyay S and Kaul-Ghanekar R: Aqueous cinnamon extract (ACE-C) from the bark of Cinnamomum cassia causes apoptosis in human cervical cancer cell line (Siha) through loss of mitochondrial membrane potential. BMC Cancer 10: 210, 2010. PMID: 20482751. DOI: 10.1186/1471-2407-10-210

13 Chen Q, Liu XF and Zheng PS: Grape seed proanthocyanidins (GSPs) inhibit the growth of cervical cancer by inducing apoptosis mediated by the mitochondrial pathway. PLoS One 9(9): e107045, 2014. PMID: 25187959. DOI: 10.1371/ journal.pone. 0107045

14 Kwon T, Chandimali N, Huynh DL, Zhang JJ, Kim N, Bak Y, Yoon DY, Yu DY, Lee JC, Gera M, Ghosh M, Park YH and Jeong DK: BRM270 inhibits cancer stem cell maintenance via microRNA regulation in chemoresistant A549 lung adenocarcinoma cells. Cell Death Dis 9(2): 244, 2018. PMID: 29445170. DOI: 10.1038/s41419-018-0277-7

15 Huynh DL, Chandimali N, Zhang JJ, Kim N, Park YH, Kwon T and Jeong DK: The novel nutraceutical KJS018A prevents hepatocarcinogenesis promoted by inflammation. Evid Based Complement Alternat Med 2018: 3909434, 2018. PMID: 30154906. DOI: $10.1155 / 2018 / 3909434$

16 Kwon T, Bak Y, Ham SY, Yu DY and Yoon DY: A1E reduces stemness and self-renewal in HPV 16-positive cervical cancer stem cells. BMC Complement Altern Med 16: 42, 2016. PMID: 26832364. DOI: 10.1186/s12906-016-1013-4
17 Kwon T, Bak Y, Park YH, Jang GB, Nam JS, Yoo JE, Park YN, Bak IS, Kim JM, Yoon DY and Yu DY: Peroxiredoxin II is essential for maintaining stemness by redox regulation in liver cancer cells. Stem Cells 34(5): 1188-1197, 2016. PMID: 26832364. DOI: $10.1186 / \mathrm{s} 12906-016-1013-4$

$18 \mathrm{Ji} \mathrm{J}$ and Zheng PS: Expression of SOX2 in human cervical carcinogenesis. Hum Pathol 41(10): 1438-1447, 2010. PMID: 20709360. DOI: 10.1016/j.humpath.2009.11.021

19 Cao L, Zhou Y, Zhai B, Liao J, Xu W, Zhang R, Li J, Zhang Y, Chen L, Qian H, Wu M and Yin Z: Sphere-forming cell subpopulations with cancer stem cell properties in human hepatoma cell lines. BMC Gastroenterol 11: 71, 2011. PMID: 21669008. DOI: 10.1186/1471-230X-11-71

20 Kabashima A, Higuchi H, Takaishi H, Matsuzaki Y, Suzuki S, Izumiya M, Iizuka H, Sakai G, Hozawa S, Azuma T and Hibi T: Side population of pancreatic cancer cells predominates in TGFbeta-mediated epithelial to mesenchymal transition and invasion. Int J Cancer 124(12): 2771-2779, 2009. PMID: 19296540. DOI: $10.1002 / \mathrm{ijc} .24349$

21 Fadare O, Reddy H, Wang J, Hileeto D, Schwartz PE and Zheng $\mathrm{W}$ : E-cadherin and beta-catenin expression in early stage cervical carcinoma: A tissue microarray study of 147 cases. World J Surg Oncol 3(38), 2005. PMID: 15969753. DOI: 10.1186/1477-78193-38

22 Vincent Z, Urakami K, Maruyama K, Yamaguchi K and Kusuhara M: CD133-positive cancer stem cells from Colo205 human colon adenocarcinoma cell line show resistance to chemotherapy and display a specific metabolomic profile. Genes Cancer 5(7-8): 250260, 2014. PMID: 25221643. DOI: 10.18632/genesandcancer.23

23 Wang L, Guo H, Lin C, Yang L and Wang X: Enrichment and characterization of cancer stemlike cells from a cervical cancer cell line. Mol Med Rep 9(6): 2117-2123, 2014. PMID: 24676900. DOI: 10.3892/mmr.2014.2063

24 O'Connor ML, Xiang D, Shigdar S, Macdonald J, Li Y, Wang T, Pu C, Wang Z, Qiao L and Duan W: Cancer stem cells: A contentious hypothesis now moving forward. Cancer Lett 344(2): 180-187, 2014. PMID: 24333726. DOI: 10.1016/j.canlet. 2013.11.012

25 Visvader JE and Lindeman GJ: Cancer stem cells: Current status and evolving complexities. Cell Stem Cell 10(6): 717-728, 2012. PMID: 22704512. DOI: 10.1016/j.stem.2012.05.007

26 Lundberg IV, Edin S, Eklof V, Oberg A, Palmqvist R and Wikberg ML: SOX2 expression is associated with a cancer stem cell state and down-regulation of $\mathrm{CDX} 2$ in colorectal cancer. BMC Cancer 16(471), 2016. PMID: 27411517. DOI: 10.1186/s12885-016-2509-5

27 Signore M, Ricci-Vitiani L and De Maria R: Targeting apoptosis pathways in cancer stem cells. Cancer Lett 332(2): 374-382, 2013. PMID: 21315505. DOI: 10.1016/j.canlet.2011.01.013

28 Scheel C and Weinberg RA: Cancer stem cells and epithelialmesenchymal transition: Concepts and molecular links. Semin Cancer Biol 22(5-6): 396-403, 2012. PMID: 22554795. DOI: 10.1016/j.semcancer.2012.04.001

Received January 20, 2020

Revised February 10, 2020 Accepted February 21, 2020 